Antônio Prates Caldeira

Gizele Carmem Fagundes

Gabriel Nobre de Aguiar

Departamento de Saúde da Mulher e da Criança. Centro de Ciências Biológicas e da Saúde. Universidade Estadual de Montes Claros. Montes Claros, MG, Brasil

Correspondência | Correspondence: Antônio Prates Caldeira

Departamento de Saúde da Mulher e da Criança

Centro de Ciências Biológicas e da Saúde Universidade Estadual de Montes Claros

Av. Dr. Ruy Braga, s/n; Vila Mauricéia 39401-089 Montes Claros, MG, Brasil E-mail: antonio.caldeira@unimontes.br

\section{Intervenção educacional em equipes do Programa de Saúde da Família para promoção da amamentação}

\author{
Educational intervention on \\ breastfeeding promotion to the \\ Family Health Program team
}

RESUMO

OBJETIVO: A iniciativa Unidade Básica Amiga da Amamentação representa um conjunto de atividades educativas dirigidas às unidades básicas de saúde. O objetivo do estudo foi avaliar a efetividade desta estratégia de promoção do aleitamento materno dirigida às equipes do Programa de Saúde da Família.

MÉTODOS: Conduziu-se um estudo de intervenção controlado com 20 equipes do Programa de Saúde da Família, selecionadas aleatoriamente em Montes Claros (MG) em 2006. O grupo sob intervenção realizou programa de treinamento específico de 24 horas para a promoção do aleitamento materno segundo a "Iniciativa Hospital Amigo da Criança". Enfatizou-se a assistência do profissional de saúde no suporte à amamentação e no manejo dos principais problemas da lactação. O grupo controle recebeu orientações habituais sobre aleitamento materno. As mães de todas as crianças menores de dois anos de idade assistidas pelas equipes foram entrevistadas antes $(\mathrm{n}=1.423)$ e 12 meses após a intervenção $(\mathrm{n}=1.491)$ e responderam questões sobre a prática da amamentação. Curvas de sobrevida do aleitamento materno foram construídas e comparadas para os dois momentos por meio do teste log rank.

RESULTADOS: Houve aumento significativo no aleitamento materno exclusivo após atividades educativas voltadas às equipes de Saúde da Família. As curvas de sobrevidas para o aleitamento materno exclusivo no primeiro momento não mostraram diferença estatisticamente significativa entre as mães assistidas por ambos os grupos $(\mathrm{p}=0,502)$. Após a intervenção, as curvas de sobrevida para o aleitamento materno exclusivo mostraram-se significativamente diferentes $(\mathrm{p}=0,001)$.

CONCLUSÕES: O treinamento das equipes de Saúde da Família da forma como propõe a Iniciativa Unidade Básica Amiga da Amamentação mostrou ser uma estratégia efetiva e de baixo custo para sensibilizar esses profissionais, uniformizando as informações e assegurando o apoio necessário para as mães com dificuldades para amamentarem seus filhos.

DESCRITORES: Aleitamento Materno. Educação em Saúde. Recursos Humanos em Saúde. Programa Saúde da Família. Promoção da Saúde. 


\begin{abstract}
OBJECTIVE: Breastfeeding Friendly Primary Care Initiative comprises educational activities focused on primary care units. The To evaluate the effectiveness of a strategy on breastfeeding promotion to the Family Health Program team.
\end{abstract}

METHODS: A controlled intervention study was performed with 20 family health care teams randomly selected into intervention and control group in Montes Claros, Southeastern Brazil, in 2006. The teams randomly selected into intervention and control group, and the intervention group took part in a 24-hour training program on breastfeeding promotion for health providers, modeled on the Baby-Friendly Hospital Initiative. It was emphasized health provider's support for breastfeeding and management of major lactation problems. The control group received routine breastfeeding training. Mothers of all children under two cared by the teams were interviewed at home before $(n=1,423)$ and 12 months after the intervention $(n=1,491)$ and answered questions about breastfeeding practices. Survival curves of breastfeeding were plotted and compared for both time points studied using the log rank test.

RESULTS: There was a significant increase in exclusive breastfeeding after the educational activities for the Family Health Program teams. Survival curves of exclusive breastfeeding at the first time point studied showed no statistical significance difference between the groups by log rank test ( $\mathrm{p}=0.502)$. After the intervention, survival curves of exclusive breastfeeding were significantly different by the log rank test ( $\mathrm{p}=0.001$ ).

CONCLUSIONS: The training of Family Health Program teams as proposed by the Baby-Friendly Hospital Initiative proved to be an effective, low-cost strategy for raising awareness among health providers, providing consistent information, and assuring the required support to mothers with breastfeeding issues.

DESCRIPTORS: Breast Feeding. Health Education. Health Manpower. Family Health Program. Health Promotion.

\title{
INTRODUÇÃO
}

O leite materno representa o melhor alimento para a criança nos primeiros meses de vida..$^{1-3}$ Contudo, apesar da excelência do aleitamento materno e da retomada da prática nos últimos anos, o desmame precoce ainda é bastante freqüente e os índices de aleitamento observados são inferiores às recomendações oficiais. ${ }^{23}$ A amamentação é uma opção materna que envolve uma complexa interação de fatores socioeconômicos, culturais e psicológicos. Os serviços materno-infantis também têm importante papel em sua promoção. Considerando o papel protetor do aleitamento materno sobre a morbidade e mortalidade infantis, as iniciativas de promoção da prática devem ser consideradas prioritárias dentro das políticas de saúde pública de cuidado infantil. ${ }^{5} \mathrm{O}$ treinamento específico é fundamental para a efetividade do trabalho de promoção da amamentação, propiciando confiança nas equipes de saúde e facilitando maior envolvimento nas atividades. ${ }^{17,18,20}$

A Iniciativa Hospital Amigo da Criança, proposta pela
Organização Mundial de Saúde (OMS), representa um marco fundamental em relação à promoção do aleitamento materno com efetiva participação dos serviços de saúde. ${ }^{22} \mathrm{~A}$ estratégia tem sensibilizado e mobilizado os profissionais de saúde na área hospitalar no sentido de uma verdadeira mudança de postura em prol do aleitamento materno. Questiona-se, todavia, o alcance das medidas adotadas nas instituições hospitalares sem um apoio efetivo das unidades básicas de saúde.

Considerando-se o potencial de promoção do aleitamento materno de tais unidades e fundamentando-se na iniciativa da OMS, o Ministério da Saúde criou a Iniciativa Unidade Básica Amiga da Amamentação (IUBAAM). A iniciativa tem por objetivo a promoção, proteção e apoio ao aleitamento materno por meio da mobilização das equipes de cuidado primário para a adoção dos "Dez Passos para o Sucesso da Amamentação”, de forma similar à Iniciativa Hospital Amigo da criança. Os passos propostos são fruto de uma revisão 
sistemática sobre as intervenções conduzidas nas fases de pré-natal e acompanhamento do binômio mãe-bebê que foram efetivas em estender a duração da amamentação. ${ }^{14}$ Foi criada também uma metodologia específica para capacitação das equipes e avaliação das unidades após treinamento. ${ }^{15}$ A proposta define o papel de suporte que as unidades básicas de saúde, em conjunto com os hospitais, podem desempenhar para tornar o aleitamento materno uma prática universal, contribuindo significativamente para a saúde e bem-estar dos bebês, suas mães, famílias e comunidade local. ${ }^{11,13}$

Como hábito alimentar, a amamentação está intrinsecamente ligada ao desenvolvimento e aos padrões culturais de uma determinada população. Esse fato justifica a necessidade de estudos e estratégias regionais que permitam atuação mais eficaz de medidas de intervenção, a partir do conhecimento da realidade local. Considerando que a promoção do aleitamento materno representa uma ação primordial para as equipes do Programa de Saúde da Família, o presente estudo teve como objetivo avaliar a efetividade da proposta educacional da IUBAAM em unidades de saúde da família.

\section{MÉTODOS}

O estudo foi desenvolvido em Montes Claros, cidade de porte médio ao norte do estado de Minas Gerais. Existem nessa cidade três hospitais credenciados pela Iniciativa Hospital Amigo da Criança, nos quais são realizados quase a totalidade dos partos locais. Na ocasião da coleta de dados, a rede básica de assistência era constituída por 15 centros de saúde e 35 unidades do Programa de Saúde da Família.

Trata-se de estudo de intervenção aleatorizado e controlado, cujo objeto foi a Unidade Básica do Programa de Saúde da Família. A intervenção proposta foi capacitar as equipes na promoção do aleitamento materno, seguindo as normas do Ministério da Saúde para o credenciamento de tais unidades como IUBAAM. Essa proposta educacional consiste de um curso de 24 horas ao longo de uma semana, incluindo atividades interativas teórico-práticas.

Todas as unidades do Programa de Saúde da Família, efetivamente ativas e completas, foram eletivas. Entre essas unidades, 20 foram recrutadas aleatoriamente, definindo-se metade delas para “intervenção” e metade como "controle".

Antes do início das atividades educativas, foram realizadas entrevistas com todas as mães de crianças menores de dois anos de idade residentes nas áreas de abrangência das unidades de saúde selecionadas. O questionário utilizado nas entrevistas com as mães era semi-estruturado, com perguntas que ensejavam respostas curtas e rápidas. A equipe de entrevistadores foi formada a partir de seleção entre estudantes da graduação do curso de medicina, especialmente treinada para os procedimentos. Tal entrevista abordou, além da história da amamentação, variáveis referentes à história obstétrica e neonatal, socioeconômicas e demográficas. Em relação ao aleitamento materno, as questões buscaram, de forma detalhada, a introdução de água, sucos, chás e outros alimentos, permitindo a definição clara do padrão alimentar em cada idade e criança investigada. Foi possível construir assim, uma análise prospectiva, a partir dos dados coletados transversalmente.

Assumindo-se já o primeiro passo da IUBAAM, as equipes para intervenção foram apresentadas a uma norma escrita, adaptada a cada uma delas e construída de maneira conjunta com a equipe de trabalho. Todas as equipes receberam a capacitação para a promoção do aleitamento materno segundo recomendações do Ministério da Saúde. O treinamento aconteceu em horário de trabalho, no Banco de Leite Humano do município, local adequado às atividades teóricas e práticas do curso, por equipe multiprofissional (pediatra, ginecologista, enfermeiro, psicólogo e assistente social, além da equipe de técnicos de enfermagem da instituição). Foram realizadas duas visitas às unidades de saúde, com discussão das principais dúvidas e orientações sobre atividades educativas. A partir da segunda visita, todas as unidades treinadas foram “credenciadas” como Unidades Básicas Amigas da Amamentação. O grupo controle não recebeu orientações ou treinamentos específicos sobre aleitamento materno no mesmo período.

Decorridos 12 meses do treinamento, todas as unidades alocadas no primeiro momento foram novamente abordadas. Entrevistas com as mães foram novamente realizadas, permitindo análise comparativa e avaliação de impacto da intervenção. Todas as informações coletadas foram codificadas e digitadas. A prevalência e a duração do aleitamento materno foram obtidas por meio de análise de sobrevida. A comparação entre as curvas de sobrevidas antes e após a intervenção foi realizada utilizando-se o teste log rank. Definiu-se em aleitamento materno todas as crianças que faziam uso do leite materno, independentemente do uso concomitante de outros alimentos. As crianças alimentadas somente com leite materno, não sendo admitido o uso de chás, água ou qualquer outro alimento foram classificadas “em aleitamento exclusivo”. O aleitamento materno predominante foi designado para os lactentes que faziam uso de leite materno em associação com chás, água e/ou sucos. Considerou-se aleitamento parcial quando havia associação com outros leites ou alimentos. Foram classificadas como desmamadas as crianças que tinham cessado completamente o uso do leite materno.

O projeto foi aprovado pelo Comitê de Ética em Pesquisa da Universidade Estadual de Montes Claros. 


\section{RESULTADOS}

Participaram do processo educacional 96 profissionais de saúde, sendo 20 entre médicos e enfermeiros, e 76 profissionais de nível médio (auxiliares e agentes comunitários de saúde). Foram realizadas entrevistas antes das intervenções educativas, com 1.423 mães de crianças identificadas na faixa etária de zero a dois anos de idade residentes e cadastradas nas equipes de saúde da família (intervenção e controle). O grupo se caracterizou como sendo de população carente, com limitado acesso aos bens de consumo e serviços em geral. Não foram observadas diferenças sociodemográficas entre o grupo controle e o grupo intervenção (Tabela).

Após 12 meses da realização das atividades educacionais, 1.491 mães de crianças menores de dois anos de idade foram entrevistadas nos mesmos serviços de saúde. Em ambos os inquéritos, as perdas ou recusas foram desprezíveis, pois todas as crianças cadastradas nas equipes foram contatadas com auxílio do agente comunitário de saúde. As variáveis sociodemográficas também não se mostraram diferentes entre os dois grupos.
As curvas de sobrevida para o aleitamento materno exclusivo no momento anterior à introdução da iniciativa são apresentadas na Figura 1. O teste log rank não mostrou diferença estatisticamente significativa entre as curvas de ambos os grupos nesse momento $(\mathrm{p}=0,502)$.

As curvas de sobrevida para o aleitamento materno exclusivo após um ano de adoção da iniciativa são apresentadas na Figura 2. O teste log rank mostrou diferença estatisticamente significativa entre as curvas dos dois grupos nesse momento $(\mathrm{p}=0,001)$. Todavia, observando-se o início das curvas em ambos as Figuras, a prática do aleitamento materno exclusivo não foi universal ao nascimento para nenhum dos grupos estudados.

A duração mediana do aleitamento materno exclusivo era de 106 dias no grupo controle no primeiro momento e passou para 107 dias um ano após. No grupo de mães assistidas pelas equipes que foram capacitadas, a duração mediana da amamentação exclusiva passou de 104 dias para 125 dias, acréscimo estatisticamente significativo.

Tabela. Características sociodemográficas de mães e crianças assistidas pela Estratégia de Saúde da Família, segundo grupos intervenção educativa e controle. Montes Claros, MG, 2006.

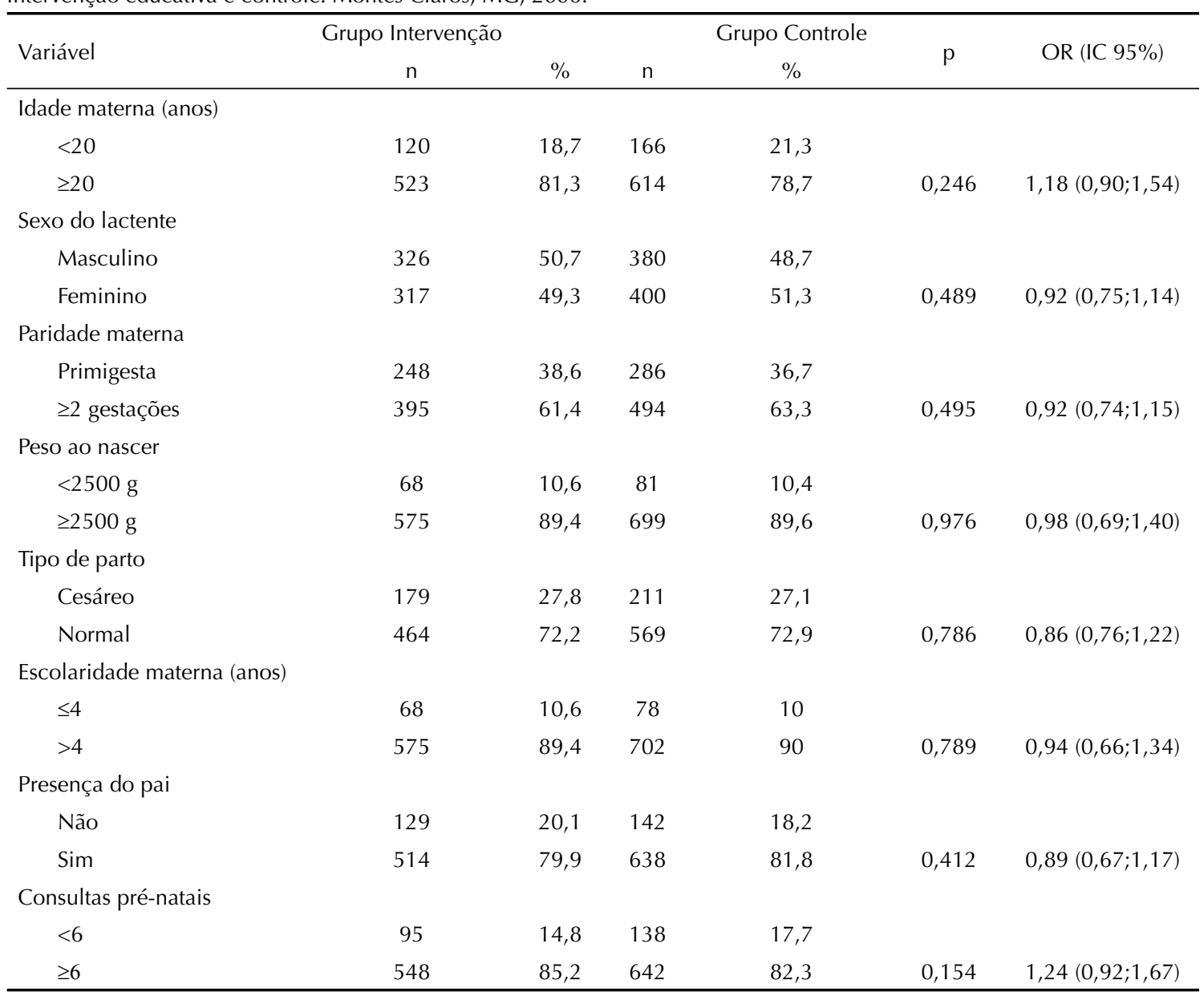




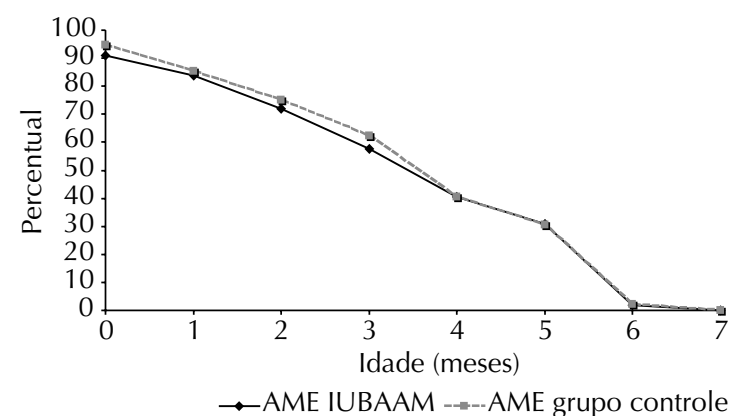

AME: Aleitamento materno exclusivo

IUBAAM: Iniciativa Unidade Básica Amiga da Amamentação

Figura 1. Curvas de sobrevida do aleitamento materno exclusivo para grupos intervenção e controle antes do treinamento. Montes Claros, MG, 2006.

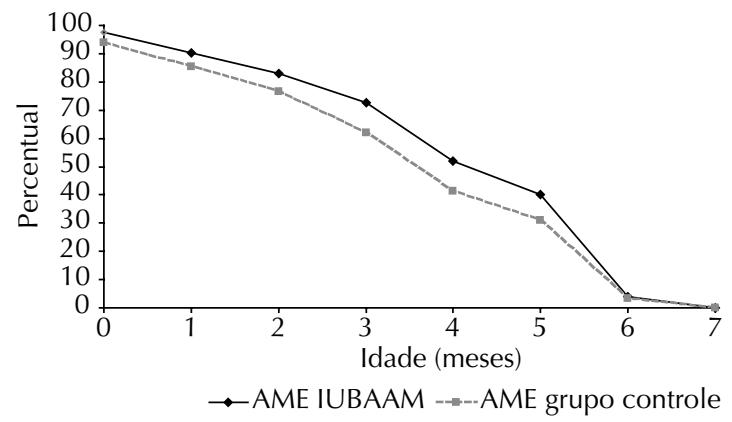

AME: Aleitamento materno exclusivo IUBAAM: Iniciativa Unidade Básica Amiga da Amamentação

Figura 2. Curvas de sobrevida do aleitamento materno exclusivo para grupos intervenção e controle após o treinamento. Montes Claros, MG, 2006.

Não foram observadas diferenças estatisticamente significativas para a duração do aleitamento materno em geral ou para o aleitamento materno predominante entre os dois grupos antes e após a intervenção.

\section{DISCUSSÃO}

Apesar de registros recentes na literatura científica de aumento dos índices de aleitamento materno, as condições ideais ainda estão distantes de serem alcançadas e justificam estudos como este, que resgatam medidas de baixo custo na promoção da prática da amamentação. ${ }^{21}$ Atividades de promoção, apoio e proteção ao aleitamento materno representam importantes estratégias para redução da morbidade infantil, especialmente em países em desenvolvimento ${ }^{5,12}$

A interpretação dos resultados do presente estudo deve tomar como limitação o local de realização, onde os dados observados já apontavam uma situação mais favorável dos indicadores de aleitamento materno desde o momento inicial. A duração mediana do aleitamento materno exclusivo foi superior a três meses em ambos os grupos, enquanto estudos nacionais não mostram tais indicadores. ${ }^{4,9,10,19}$ Os resultados locais representam uma particularidade da cidade, que conta com três hospitais "amigos da criança", que realizam mais de 97\% dos partos do município e têm relevante impacto nos indicadores de aleitamento materno do município.7 Essa situação favorece a cultura do aleitamento materno, com início precoce da amamentação na sala de parto, incentivo ao aleitamento materno sob livre demanda no alojamento conjunto e desestímulo ao uso de utensílios que favoreçam o desmame, além das atividades educativas de promoção da prática que são desenvolvidas nos hospitais da Iniciativa Hospital Amigo da Criança. ${ }^{22}$

Outra possível limitação é de que no presente estudo somente foram alocadas as equipes da área urbana. Contudo, essa restrição logística foi fundamentada em evidências de que a complexidade da equipe do cuidado primário ou o seu local de inserção não interfere nos resultados de programas educacionais de promoção do aleitamento materno, conforme apontaram estudos anteriores. ${ }^{14,15}$

Não houve, no presente estudo, aferição contínua e direta dos conhecimentos dos profissionais de saúde após participação nas atividades educativas. Essa aferição foi limitada ao pós-teste incluído no processo de capacitação. Contudo, estudos de avaliação e habilidades após treinamentos específicos já mostraram efetividade da capacitação. ${ }^{14,17,20} \mathrm{O}$ presente estudo apresenta outro enfoque no processo de avaliação de estratégias educacionais para profissionais do cuidado primário, sendo dirigida aos resultados ou impactos na comunidade assistida. As equipes de saúde da família ostentam uma condição privilegiada para atividades afins. A inserção na comunidade, o conhecimento dos mitos e tabus locais sobre o tema e o envolvimento de lideranças locais, representadas pelos agentes comunitários de saúde, propiciam maiores chances de êxito para atividades educativas. ${ }^{16}$ Porém, é preciso oferecer a esses atores sociais o conhecimento adequado que os proporcione segurança na transmissão de informações e os habilite para apoiar e oferecer o suporte necessário para as mães com dificuldades para amamentar. Estudo prévio local mostrou que embora esses profissionais possuam a informação sobre as vantagens do aleitamento materno, eles não dispõem de informações para manejo das principais dificuldades enfrentadas pelas nutrizes, como ingurgitamento, técnica inadequada, ou problemas com os mamilos. ${ }^{6}$

A participação dos agentes comunitários de saúde parece ter sido fundamental no sucesso da iniciativa, pois são mais envolvidos com a comunidade e com atividades de promoção do aleitamento materno em suas funções habituais, enquanto médicos e enfermeiros referem 
menos oportunidades práticas de intervenção. ${ }^{6}$ Segundo a literatura, a participação de pessoas leigas, como os agentes comunitários, no processo de aconselhamento parece ser mais efetiva para aumentar a duração do aleitamento materno exclusivo, enquanto os profissionais de saúde parecem ser mais efetivos em estender a duração do aleitamento materno em geral. ${ }^{21}$

O fato de não ter sido observada interferência significativa da Iniciativa sobre os indicadores de aleitamento materno predominante ou aleitamento materno em geral pode ser decorrente do pouco tempo de observação (apenas um ano) ou ser, realmente uma limitação das estratégias educativas, pelo menos para populações semelhantes, com indicadores mais próximos dos desejáveis. Novos estudos são necessários para averiguar tais questões.

Concluindo, o treinamento das equipes de Saúde da Família da forma como propõe a Iniciativa Unidade Básica Amiga da Amamentação parece ser uma estratégia efetiva e de baixo custo para sensibilizar esses profissionais, uniformizando as informações e assegurando o apoio necessário para as mães com dificuldades para amamentarem seus filhos. As equipes de saúde da família atuam com real envolvimento da comunidade por meio dos agentes comunitários de saúde. Existe, assim, uma tendência natural à construção de uma rede de suporte e apoio à prática da amamentação, com modificação gradual da cultura local. Estudo prévio já mostrou a efetividade do suporte domiciliar. ${ }^{8}$ Durante alguns anos, os treinamentos na área de aleitamento materno foram negligenciados pelos profissionais de saúde em geral, o que gerou uma falta de informações e habilidades aos profissionais. ${ }^{21}$ Ainda que reconheçam a importância da prática, quase sempre faltam a esses profissionais o conhecimento técnico para abordar questões práticas como a adequação da pega, o ingurgitamento, as fissuras entre outros problemas. ${ }^{6}$ Os resultados do presente estudo recomendam ampla divulgação da Iniciativa Unidade Básica Amiga da Amamentação para as equipes de Saúde da Família, particularmente para as áreas onde ainda existam elevados coeficientes de morbidade e mortalidade infantis. Reitera-se, contudo, a necessidade de apoio e vigilância às equipes capacitadas para que se mantenham seguras em suas habilidades e conhecimentos para contínua promoção do aleitamento materno. 


\section{REFERÊNCIAS}

1. American Academy of Pediatrics, Work Group on Breastfeeding. Breastfeeding and the use of human milk. Pediatrics. 1997;100(6):1035-9. DOI: 10.1542/ peds.100.6.1035

2. Anderson JW, Johnstone BM, Remely DT. Breastfeeding and cognitive development: a meta-analysis. Am J Clin Nutr. 1999;70(4):525-35.

3. Bachrach VR, Schwarz E, Bachrach LR. Breastfeeding and the risk of hospitalization for respiratory disease in infancy: a meta-analysis. Arch Pediatr Adolesc Med. 2003;157(3):237-43.

4. Bittencourt LJ, Oliveira JS, Figueiroa JN, Batista Filho M. Aleitamento materno no estado de Pernambuco: prevalência e possível papel das ações de saúde. Rev Bras Saude Matern Infant. 2005;5(4):439-48. DOI: 10.1590/S1519-38292005000400007

5. Black RE, Morris SS, Bryce J. Where and why are 10 million children dying every year? Lancet. 2003;361(9376):2226-34. DOI: 10.1016/S01406736(03)13779-8

6. Caldeira AP, Aguiar GN, Magalhães WAC, Fagundes GC. Conhecimentos e práticas de promoção do aleitamento materno em Equipes de Saúde da Família em Montes Claros, Minas Gerais, Brasil. Cad Saude Publica. 2007;23(8):1965-70. DOI: 10.1590/S0102311 X2007000800023

7. Caldeira AP, Gonçalves E. Assessment of the impact of implementing the Baby-Friendly Hospital Initiative. J Pediatr (Rio J). 2007;83(2):127-32. DOI: 10.1590/ S0021-75572007000200006

8. Coutinho SB, Lira PIC, Lima MC, Ashworth A. Comparison of the effect of two systems for the promotion of exclusive breastfeeding. Lancet. 2005;366(9491):1094-100. DOI: 10.1016/S01406736(05)67421-1

9. Del Ciampo LA, Junqueira MJG, Ricco RG, Daneluzzi JC, Ferraz IS, Martinelli Jr CE. Tendência secular do aleitamento materno em uma unidade de atenção primária à saúde materno-infantil em Ribeirão Preto, São Paulo. Rev Bras Saude Matern Infant. 2006;6(4):391-96. DOI: 10.1590/S151938292006000400005

10. Faleiros JJ, Kalil G, Casarin DP, Laque Jr PA, Santos IS. Avaliação do impacto de um programa de puericultura na promoção da amamentação exclusiva. Cad Saude Publica. 2005;21(2):482-89. DOI: 10.1590/S0102311 X2005000200014

11. Guise JM, Palda V, Westhoff C, Chan B, Helfand M. Lieu TA, et al. The effectiveness of primary care-based interventions to promote breastfeeding: systematic evidence review and meta-analysis for the US Preventive Services Task Force. Ann Fam Med. 2003;1(2):70-8. DOI: 10.1370/afm.56
12. Jones G, Steketee RW, Black RE, Bhutta ZA, Morris SS, Bellagio Child Survival Study Group. How many child deaths can we prevent this year? Lancet. 2003;362(9377):65-71. DOI: 10.1016/S01406736(03)13811-1

13. Lana APB, Lamounier JA, César CC. Impacto de um programa para promoção da amamentação em um centro de saúde. J Pediatr (Rio J). 2004;80(3):235-40. DOI: 10.1590/S0021-75572004000400013

14. Oliveira MIC, Camacho LAB. Impacto das unidades básicas de saúde na duração do aleitamento materno exclusivo. Rev Bras Epidemiol. 2002;5(1):41-51. DOI: 10.1590/S1415-790X2002000100006

15. Oliveira MI, Camacho LAB, Tedstone, AE. Extending breastfeeding duration through primary care: a systematic review of prenatal and postnatal interventions. J Hum Lact. 2001;17(4):326-43. DOI: $10.1177 / 089033440101700407$

16. Parada CMGL, Carvalhaes MABL, Winckler CC, Winckler LA, Winckler VC. Situação do aleitamento materno em população assistida pelo programa de saúde da família-PSF. Rev Latino-am Enfermagem. 2005;13(3):407-14. DOI: 10.1590/S010411692005000300016

17. Rea MF, Venâncio SI, Martines JC, Savage F. Counselling on breastfeeding: assessing knowledge and skills. Bull World Health Organ. 1999;77(6):492-8.

18. Rodríguez-García R, Schaefer LA. Nuevos Conceptos de lactancia, su promoción y la educación de los profesionales de la salud. Bol Oficina Sanit Panam. 1991;111(1):1-14.

19. Silveira FJF, Lamounier JA. Prevalência do aleitamento materno e práticas de alimentação complementar em crianças com até 24 meses de idade na região do Alto Jequitinhonha, Minas Gerais. Rev Nutr. 2004;17(4):437-47. DOI: 10.1590/S141552732004000400004

20. Valdés V, Pugin E, Labbok MH, Pérez A, Catalán S, Aravena R, Adler MR. The effects in professional practices of a three-day course on breastfeeding. I Hum Lact. 1995;11(3):185-90. DOI: 10.1177/0890334 49501100318

21. World Health Organization. Community-based strategies for breastfeeding promotion and support in developing countries. Geneva; 2003.

22. World Health Organization. Division of Child Health and Development. Evidence for the Ten Steps to Successful Breastfeeding. Geneva; 1998.

23. World Health Organization. Global Strategy on Infant and Young Child Feeding. 55th World Health Assembly. Geneva; 2002. 